\title{
Reglindis Böhm, Präsidentin des Landgerichts Kassel i.R.
}

\author{
Juristinnen machen Karriere - wir stellen sie vor
}

Das Gespräch mit Reglindis Böhm führte Antje Sedemund-Treiber, Präsidentin des BPatG i.R. und Ehrenpräsidentin des djb, im Dezember 2008.

Frau Böhm, Sie haben einen für eine Frau Ihrer Generation außergewöhnlichen Berufsweg absolviert. Ist der Berufsweg der Juristin bereits in Ihrem Elternhaus angelegt worden oder hat er sich auf andere Weise ergeben?

Mein späterer Beruf war durch mein Elternhaus nicht vorgezeichnet. Meine Mutter war promovierte Kunsthistorikerin, mein Vater als Lehrer mit dem Schwerpunkt Geschichte ebenfalls geisteswissenschaftlich ausgerichtet. Besonderen Einfluss auf mich hatte mein Großvater mütterlicherseits. Meine Eltern trennten sich früh; unmittelbar nach Kriegsende wurde ihre Ehe geschieden. Meine Mutter zog mit meiner Schwester und mir zu ihren Eltern nach Bad Nauheim. Mein Großvater war als Direktor der Augustinerschule in Friedberg bereits 1934 aus dem Schuldienst entlassen worden, weil er sich weigerte, der NSDAP beizutreten; darüber hinaus war er mit kritischen Äußerungen aufgefallen. Das Fehlen einer beruflichen Aufgabe überbrückte mein Großvater mit Geigen- und Klavierspiel; er war sehr musikalisch. Unter seinem Einfluss habe ich früh begonnen, Geige zu spielen und übte bis zu fünf Stunden am Tag. Dabei entwickelte sich auch mein erster Berufswunsch: Ich wollte Geigerin werden.

Im Ergebnis habe ich dieses Ziel dann aber nicht weiterverfolgt. Ich wollte als Musikerin nicht in den Schuldienst; auch in einem Orchester wollte ich nicht spielen. Für eine Solistin aber reichte meine Begabung nicht. ich musizierte häufig mit einem früheren Mitschüler. Er hatte Jura studiert und empfahl mir, mich einmal darüber zu informieren. Ich tat dies, fand Gefallen an der Materie und kam überraschend gut zurecht. Außerdem war das juristische Studium kurz und damit in seiner Finanzierung kalkulierbar. Das war aus wirtschaftlichen Gründen sehr wichtig für mich, ich musste mein Studium weitgehend selbst finanzieren. Ich übernahm deshalb nebenher die verschiedensten Arbeiten, in Fabriken, im Büro und auch im Haushalt. Dort waren meine Leistungen allerdings nicht besonders gut.

Um auf Ihre Ausgangsfrage zurückzukommen: Juristin bin ich also eigentlich durch Zufall geworden. Die Musik hat mich natürlich weiter begleitet. Ich habe sie nicht nur privat ausgeübt, sondern auch bei öffentlichen Anlässen, so habe ich in der Frankfurter Kantorei gesungen und auch als Aushilfe in Orchestern gespielt.

Würden Sie denselben Berufsweg noch einmal wählen und welche Erfahrungen haben Sie dort in einer Zeit gemacht, in der Frauen vor allem auf der höheren Ebene noch eine Seltenheit waren?
Reglindis Böhm, geboren 1934, wuchs in Hessen und Rheinhessen auf. Sie studierte Rechtswissenschaften an der Johann-WolfgangGoethe Universität in Frankfurt am Main und schloss ihr Studium bereits nach dem 7. Semester mit dem ersten Staatsexamen ab. Es folgte die Referendarzeit in den Landgerichtsbezirken Frankfurt am Main und Kassel. Ihr zweites Staatsexamen legte Reglindis Böhm $1961 \mathrm{ab}$.

Reglindis Böhm begann ihren Berufsweg als Rechtsanwältin und freie Mitarbeiterin in einer Anwaltskanzlei. Anfang 1967 wechselte sie in den Staatsdienst des Landes Hessen und war zunächst am Amtsund Landgericht tätig. 1972 wurde sie zur Richterin am Oberlandesgericht Frankfurt am Main ernannt und war hier zunächst in einem Zivilsenat, danach in einem Strafsenat eingesetzt. Mit Einführung der Familiengerichtsbarkeit zum 1. Juli 1977 wurde Reglindis Böhm Beisitzende in dem neu eingerichteten Familiensenat und gleichzeitig stellvertretende Vorsitzende. Bei der Einrichtung des inzwischen vierten Familiensenats bei dem Oberlandesgericht Frankfurt am Main im Jahr 1985 wechselte Reglindis Böhm in diesen Senat und übernahm dessen Vorsitz. Von Ende 1990 bis zu ihrer Pensionierung im Jahr 1999 war Reglindis Böhm Präsidentin des Landgerichts Kassel. Als Richterin war sie in dieser Zeit Vorsitzende einer zweitinstanzlichen Zivilkammer mit den Arbeitsfeldern Vormundschaftssachen, Verwandtenunterhalt, Recht der nichtehelichen Kinder und allgemeinen Berufungen gegen Entscheidungen mehrerer Amtsgerichte.

Reglindis Böhm ist Trägerin des Bundesverdienstkreuzes am Bande des Verdienstordens der Bundesrepublik Deutschland.

Sie ist verwitwet und hat zwei erwachsene Söhne.

Um dies vorweg zu sagen: Ich habe meine Entscheidung für den Beruf der Juristin nie bedauert und würde sie in gleicher Weise wieder treffen. Schon die juristische Denkweise lag mir von Anfang an. Darüber hinaus war Frankfurt eine lebendige Universität mit vielen Anregungen, Es gab sogar eine interdisziplinäre Vereinigung, das „Collegium studentischer Club“, dem zahlreiche Studentinnen angehörten. Im Vorstand war schon damals immer eine Frau, die aktiv das Programm mitbestimmte und gestaltete. Es umfasste wissenschaftliche Vorträge verschiedener Fakultäten, Radtouren zu kulturell interessanten Orten, aber auch Feste.

Studium und Beruf haben mir aber auch inhaltlich Freude gemacht. Das Familienrecht im weiteren Sinne, das zum Mittelpunkt meiner Tätigkeit wurde, umfasst als erstes den Umgang mit Menschen; er war mir immer wichtig. Gute Lösun- 
gen in familienrechtlichen Fragen müssen darüber hinaus über die Bewältigung des konkreten Problems hinausgehen. Sie umfassen psychologische und rechtssoziologische Komponenten. Beides sind für mich grundlegende Ansatzpunkte, die mich immer sehr interessiert und beschäftigt haben. So habe ich an einer Balint-Gruppe unter Supervision einer Psychoanalytikerin teilgenommen. Das war besonders hilfreich für die Entscheidung streitiger Sorgerechtssachen, da eine gesteigerte Sensibilität für die Spannungen und Interaktionen gestörter Familien geweckt wurde. Von dieser Sicht- und Arbeitsweise her ergaben sich im Übrigen Verbindungen zur Tätigkeit meines inzwischen leider verstorbenen Mannes. Er war ebenfalls Jurist, aber mit den Schwerpunkten Strafrecht, Kriminologie und Strafvollzug. Nach der Sammlung praktischer Erfahrungen im Strafvollzug - er leitete zeitweise die Jugendstrafanstalt Rockenberg - hatte er einen Lehrstuhl an der Universität Mainz mit den genannten Schwerpunkten inne.

Im Übrigen: Frauen waren damals in der Justiz und natürlich ganz besonders bei den höheren Gerichten noch sehr selten anzutreffen. Als ich 1972 an das Oberlandesgericht Frankfurt kam, war ich die dritte Frau. Die Leitung eines Landgerichts, im konkreten Fall in Kassel, habe ich als erste Frau in Hessen übernommen. Schwierigkeiten, insbesondere mit männlichen Kollegen, habe ich nie gehabt. Mir mag dabei zugute gekommen sein, dass ich gern im Team arbeite. Das ist schon in einem kollegial besetzten Spruchkörper wichtig, gilt darüber hinaus aber auch für die Tätigkeit in einer Verwaltung wie beim Landgericht. Wichtig ist, wie man mit den Kollegen umgeht und ob man von ihnen auch Belehrungen annimmt. Frauenförderung war für mich als Behördenchefin kein Problem. Qualifizierte Frauen haben gute Chancen weiterzukommen.

Gab es weitere Bereiche oder Themen im Zusammenhang mit Ihrem Beruf, die Ihnen wichtig waren?

Drei Schwerpunkte möchte ich nennen:

Die Mitwirkung an der Aus- und Fortbildung von Juristen war mir immer wichtig. Schon während meiner Zeit als Richterin am Landgericht Gießen - ich gehörte sowohl einer Zivil- als auch einer Strafkammer an - leitete ich eine Referendararbeitsgemeinschaft. Später habe ich mich zusätzlich in der Referendarausbildung sowie bei der Fortbildung von Richtern und Rechtsanwälten im Familienrecht engagiert. Nach der Wiedervereinigung habe ich an der Aus- und Fortbildung der Juristinnen und Juristen aus der früheren DDR mit- wirken können. Es begann mit Fortbildungskursen für frühere Diplomjuristen zunächst in Berlin und dann in Thüringen; ich habe Zivilprozess- und Kaufrecht unterrichtet. Es folgte die Mitwirkung an der Referendarausbildung für Diplomjuristen aus Thüringen; sie begann am Landgericht Kassel. Schließlich haben wir die Bereichsrechtspfleger für die Bezirksgerichte Gera und Erfurt ausgebildet. Sie erinnern sich sicherlich: In der früheren DDR gab es keine Rechtspfleger. Man brauchte sie aber dringend in der Justiz nach der Wiedervereinigung, am Anfang ganz besonders bei den Grundbuchämtern und beim Handelsregister.

Wichtig war mir darüber hinaus eine vertiefte wissenschaftliche, psychologische und rechtspolitische Auseinandersetzung mit den Themen, die mich in meinem Berufsalltag beschäftigten. Dies habe ich in einer Reihe von Aufsätzen und immer wieder auch in Vorträgen getan. Die behandelten Themen waren vielfältig. Sie reichten vom Strafrecht und Strafvollzug über das Jugendhilferecht bis zu verschiedenen Fragen des Familienrechts. Auch mit der richterlichen Selbstverwaltung, so den Mitwirkungsrechten im Richterrat, und mit der Situation der Frauen in der Justiz habe ich mich befasst.

Von hier aus war es nur ein kleiner Schritt zur Mitwirkung in Fachverbänden. Außer dem djb gehöre ich seit langem dem Deutschen Verein für öffentliche und private Fürsorge an, früher in Frankfurt am Main, heute in Berlin. Ich bin dort Mitglied in dem Fachausschuss Jugend und Familie, in dem Arbeitskreis Familienpolitik sowie im Hauptausschuss. Hier habe ich an Stellungnahmen zur Reform des Kindschaftsrechts, des Jugendhilferechts und der Freiwilligen Gerichtsbarkeit mitgearbeitet; einige Jahre habe ich auch die Arbeitsgruppe Kindschaftsrechtsreform geleitet. Während meiner Kasseler Zeit war ich darüber hinaus mehrfach Vorsitzende der dortigen Juristischen Gesellschaft und im Vorstand des Gustav Radbruch-Vereins, der sich mit straffälligen Jugendlichen befasste. Ich gehöre dem Familiengerichtstag und der Wissenschaftlichen Vereinigung für Familienrecht an und war lange Jahre auch in der kirchlichen Gerichtsbarkeit tätig.

Sie waren beruflich gefordert und haben sich darüber hinaus erheblich engagiert: Wie ist es Ihnen gelungen, dies weite berufliche Feld mit den Pflichten zu vereinbaren, die in Ihrer Familie auf Sie warteten?

Ich habe relativ früh, Anfang 1959, geheiratet. Mein erster Sohn wurde schon 1960 geboren. Ich absolvierte damals gerade als Referendarin meine 
Station am Oberlandesgericht. Mein zweiter Sohn folgte dann 1963. Die praktische Versorgung der beiden Kinder haben mein Mann und ich uns nach den tatsächlich gegebenen Möglichkeiten geteilt. Organisationstalent und Flexibilität waren natürlich gefragt. So habe ich den Beginn meiner Berufstätigkeit nach der erforderlichen Betreuung meiner Kinder ausgerichtet. Da es im Staatsdienst noch keine Teilzeitarbeit für Mütter mit Kindern gab, wurde ich Rechtsanwältin und nahm stellungspraxis bei Richterinnen und Rechtspflegerinnen. Da diese häufig bessere Examensergebnisse erreichten als ihre männlichen Kollegen, mussten mehr Frauen als Männer eingestellt werden. Teilweise wurde über Gegenstrategien nachgedacht, die aber unhaltbar waren und auf breite Kritik stießen. Sie sind dann später auch tatsächlich nicht verwirklicht worden. Im Anschluss an den Vortrag von Renate Damm bin ich in den djb eingetreten.

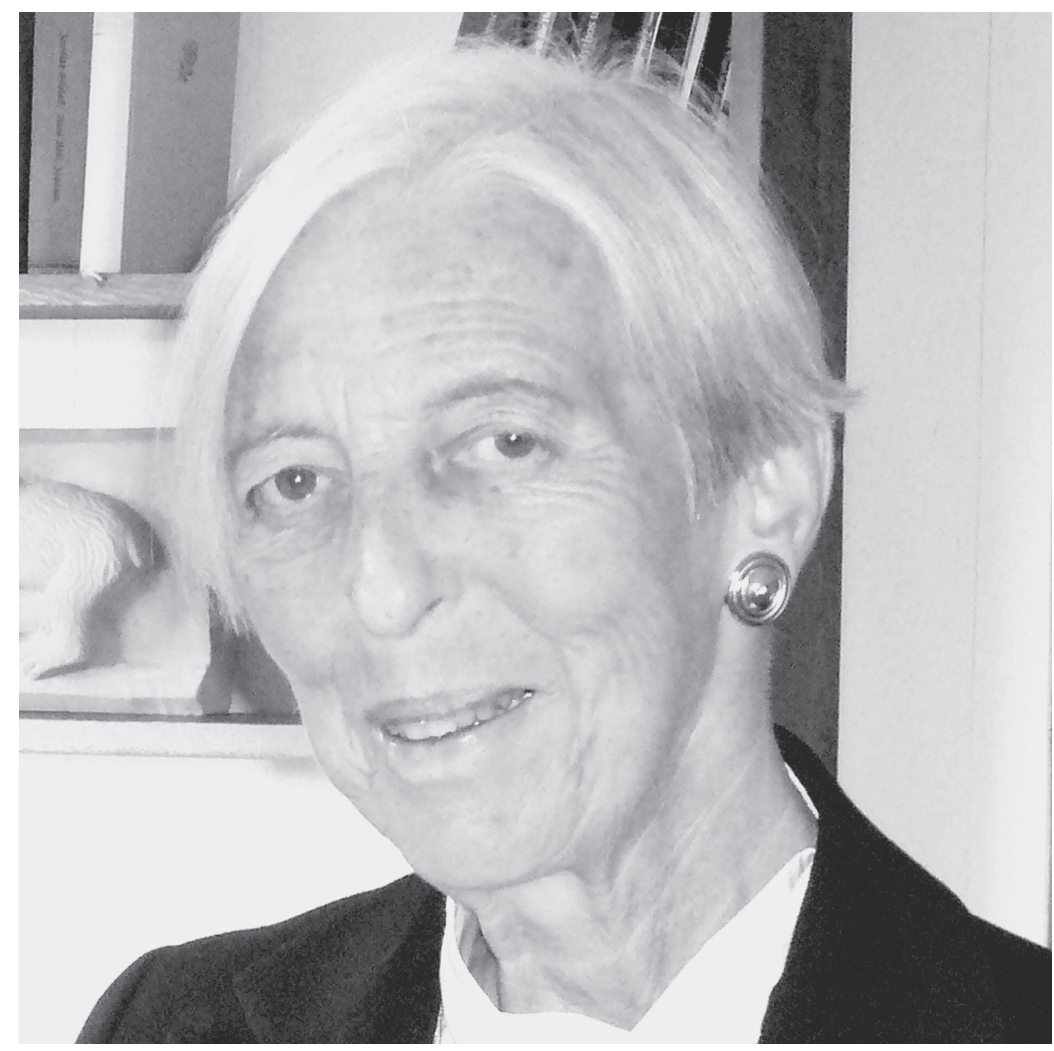

Vertretungen wahr. Zwei volle Tage in der Woche arbeitete ich im Büro und war dort präsent, die übrige Zeit zu Hause. So ließen sich zum Beispiel Berufungsbegründungen gut zu Hause anfertigen. Mein Mann hat mich bei der Erziehung und Versorgung der Kinder stets unterstützt. Ohne seine Hilfe hätte ich dies alles wohl kaum geschafft. In seiner Einstellung - auch zu meiner Berufstätigkeit - war mein Mann stark durch seine Großmutter, die Dichterin Ricarda Huch, geprägt. Sie war eine der ersten promovierten Frauen.

Was hat Sie veranlasst, Mitglied des djb zu werden und wo lagen die Schwerpunkte Ihrer Arbeit in unserem Verband?

Auf den djb aufmerksam gemacht hat mich ein Vortrag, den Renate Damm, damals Erste Vorsitzende des Verbandes, im November 1985 vor dem Hessischen Richterbund zu dem Thema „Justitia - ein Mann?“ gehalten hat. Hintergrund waren zum Teil kursierende kritische Überlegungen zur Ein-
Im djb habe ich zunächst in der Familienrechtskommission mitgearbeitet. Den Vorsitz führte damals Frau Peschel-Gutzeit; es war eine interessante und fruchtbare Zeit. Von 1991 bis 1994 habe ich dann selbst den Vorsitz der Familienrechtskommission wahrgenommen, von 1992 bis 1996 auch den der Unterkommission „Kindschaftsrecht“. Unter dem Vorsitz von Frau Schwenzer hatten wir Vorschläge für eine Gleichstellung der nichtehelichen mit den ehelichen Kindern im Familien- und Erbrecht erarbeitet. Aus der Feder von Frau Schwenzer stammte ein 1992 publizierter Gesetzentwurf zur Neuregelung des Kindschaftsrechts.

Im Übrigen gehöre ich seit einigen Jahren der von Frau Schreiber engagiert geleiteten Kommission „Ältere Menschen“ an. Ich war dort an der Neufassung der Patientenverfügung beteiligt, die uns weiterhin ebenso beschäftigt wie erforderliche Änderungen in den Heimgesetzen der Länder und das Problem der Gewalt gegen ältere Menschen. Schließlich 
war ich von 1989 bis 1991 Beisitzerin im Bundesvorstand, den Sie, Frau Sedemund-Treiber, damals als Erste Vorsitzende des Verbandes geleitet haben. Insgesamt hat mir alles große Freude gemacht. An die Zeit im Bundesvorstand erinnere ich mich besonders gern. Es war eine sehr vielseitige und lebendige Zusammenarbeit.

Wo sehen Sie gegenwärtig Schwerpunkte der Arbeit des djb und was macht unseren Verband heute für junge Juristinnen interessant?

Ein Schwerpunkt der Arbeit des djb ist aus meiner Sicht, geprägt natürlich durch meine fachliche Ausrichtung und Erfahrung, noch immer und auch zukünftig das Familienrecht. Die strukturelle Benachteiligung von Frauen ist in diesem Bereich nach wie vor evident und nicht überwunden. Ein erschreckendes Beispiel dafür ist das neue Unterhaltsrecht für geschiedene Frauen mit seinem überzogenen Grundsatz der Eigenverantwortung. Das neue Recht soll Mütter bereits vom dritten Lebensjahr eines Kindes an dazu anhalten, grundsätzlich selbst für ihren Unterhalt zu sorgen. Dieses neue Recht nimmt keine Rücksicht auf die individuell sehr unterschiedlichen Bedürfnisse der Kinder, die immer noch ungenügende Betreuungssituation und fehlende Arbeitsplätze. Eine Übergangsregelung für Altehen fehlt, obwohl diese häufig unter ganz anderen Voraussetzungen geschlossen wurden. Ich erinnere an die Stel- lungnahme der Familienrechtskommission des djb zum Unterhaltsänderungsgesetz 1986. Eine nachträgliche Begrenzung und Abschmelzung der nachehelichen Unterhaltsansprüche wird darin abgelehnt, weil solche Maßnahmen vor allem sozial schwächer gestellte, arbeitslose Frauen und Frauen mit Kindern treffen. Für wichtig halte ich auch die weitere Arbeit unseres Verbandes zum Thema „Ältere Menschen“. Für diese Gruppe unserer Mitbürger wird immer noch zu wenig getan. Heimplätze sind kaum bezahlbar, die Preise - auch im Ländervergleich - sind unterschiedlich, die Kalkulation ist nicht nachvollziehbar. Dies ist nur ein Komplex, mit dem sich unser Verband kontinuierlich befassen muss.

Von den älteren Menschen zu den jungen Kolleginnen: Der djb gibt viel und man kann dort auch viel lernen. Wichtig und interessant finde ich den djb als Stätte eines Zusammentreffens von Frauen der verschiedensten Berufszweige und Altersgruppen. Abgesehen von den persönlichen Begegnungen, die mir immer viel bedeutet und gegeben haben, trifft man auf breit gestreute und vielseitige Erfahrungen. Wichtig ist es natürlich auch, die Arbeit des Verbandes kennenzulernen. Sie führt den Blick über den Zaun, vor allem wenn man aktiv mitmacht. Als junge Juristin würde ich deshalb heute selbstverständlich - wieder - dem djb beitreten.

Frau Böhm, ich danke Ihnen für dieses Gespräch.

\section{Impressum}

\author{
Schriftleitung: \\ Anke Gimbal, Rechtsassessorin (V.i.S.d.P.) \\ Juliane Lindner \\ Redaktionsanschrift: \\ Deutscher Juristinnenbund e. V. \\ Anklamer Str. 38 \\ 10115 Berlin \\ Telefon: 030 443270-0 \\ Telefax: 030 443270-22 \\ E-Mail: geschaeftsstelle@djb.de

\section{Druck und Verlag:} \\ Nomos Verlagsgesellschaft mbH \& Co. KG \\ Waldseestr. 3-5 \\ D-76530 Baden-Baden \\ Telefon: 07221 2104-O \\ Telefax: 07221 2104-27
}

\author{
Anzeigenverwaltung und \\ Anzeigenannahme: \\ Sales friendly, Verlagsdienstleistungen \\ Bettina Roos \\ Siegburgerstr. 123 \\ D-53229 Bonn \\ Telefon: 0228 97898-o \\ Telefax: 0228 97898-20 \\ E-Mail: roos@sales-friendly.de
}

Die Zeitschrift sowie alle in ihr enthaltenen einzelnen Beiträge und Abbildungen sind urheberrechtlich geschützt. Jede Verwertung, die nicht ausdrücklich vom Urheberrechtsgesetz zugelassen ist, bedarf der vorherigen Zustimmung des Verlages.

Namentlich gekennzeichnete Artikel müssen nicht die Meinung des Herausgebers oder der Schriftleitung wiedergeben. Unverlangt eingesendete Manuskripte - für die keine Haftung übernommen wird - gelten als Veröffentli- chungsvorschlag zu den Bedingungen des Verlags. Es werden nur unveröffentlichte Originalarbeiten angenommen. Die Verfasser erklären sich mit einer nicht sinnentstellenden redaktionellen Bearbeitung einverstanden.

\author{
Erscheinungsweise: \\ vierteljährlich \\ Bezugspreis 2010: \\ jährlich 48,-€, Einzelheft 13,-€
}

Alle Preise zzgl. Vertriebs-Direktbeordnungsgebühren inkl. MWSt.;

Bestellungen nehmen entgegen:

Der Buchhandel und der Verlag; Kündigung jeweils drei Monate zum Kalenderjahresende. Zahlungen jeweils im Voraus an: Nomos Verlagsgesellschaft, Postbank Karlsruhe, Konto 7363651 (BLZ 66010075) oder Stadtsparkasse Baden-Baden, Konto 5002266 (BLZ 66250030). 